\title{
Dynamischer Prozess
}

Der produktbezogene Umweltschutz - mittlerweile zumeist „Integrierte Produktpolitik" (IPP) genannt - steht seit kurzem ganz oben auf der politischen Agenda. Deutschland nutzte seine EU-Ratspräsidentschaft, um das Thema voranzutreiben. Der nächste Schritt ist ein Grünbuch der Europäischen Kommission, das im Frühjahr 2000 erscheinen soll. Zugleich ist IPP ein Paradebeispiel für den Verlauf von Politikprozessen und die Rolle der Forschung dabei.

$\mathrm{D}_{\text {. }}$ beträchtliche Erfolge im prozess- und anlagenbezogenen Umweltschutz erzielt; mittlerweile wurde jedoch eine zunehmende Bedeutung eines produktbezogenen Ansatzes für die Umweltpolitik erkannt. Integrierte Produktpolitik (IPP) ist eine Antwort auf diese Herausforderungen. Rubik/ Simshäuser - Text

Unter IPP versteht man ,eine staatliche Politik, die auf eine stetige Verbesserung von Produkten und Dienstleistungen hinsichtlich ihrer umweltrelevanten Wirkungen entlang des gesamten Produktlebenszyklusses abzielt bzw. diese fördert." (1)

Die Entstehungsgeschichte von IPP steht für den Wandel in der Bedeutung des Umweltschutzes heraus aus einem exotischen Nischendasein hin zu einem bestimmenden Faktor für globale politische Verantwortung. Über „Umweltskandale“, öffentlichen Druck und einschlägige internationale Konferenzen (Rio etc.) entwickelte sich im Europa der 80-er Jahre auch in vielen europäischen Unternehmen eine neue Denk- und Managementkultur. Verbesserte Produkte wurden in vielen Bereichen geschaffen und belebten den Wettbewerb. Die Entwicklung von Maßnahmen, Instrumenten und ersten konzeptionellen Ansätzen der produktbezogenen Umweltpolitik erfolgte insbesondere in nordeuropäischen Ländern.

\section{Drei Entwicklungsstränge}

Aber erst in den 90er Jahren kamen die Dinge rascher voran. Drei Entwicklungsstränge können dabei unterschieden werden (2):

Forschung: Die ersten wesentlichen und systematischen Zugänge zum Thema IPP wurden von IVM und IÖW mit einem von der ForschungsGeneraldirektion der Europäischen Kommission finanzierten Projekt „Product policy in support of environmental policy“ gelegt (3). Eine Vielzahl von Arbeiten widmete sich speziellen Forschungsfragen, um das mögliche Instrumentarium einer IPP zu erarbeiten bzw. zu verbessern. Im Blickpunkt standen Untersuchungen zu Umweltkennzeichnung, Produktrücknahme und -verantwortung, öffentlichem Beschaffungswesen und Steuern/ Abgaben.

Politik: Das fünfte Umweltaktionsprogramm der Europäischen Union sprach bereits implizit IPP an; in einem Fortschrittsbericht der Kommission von 1995 wurde erstmals explizit auf die Notwendigkeit von zusätzlichen Maßnahmen im Bereich der Produktpolitik verwiesen. Einflüsse gingen auch von der „Agenda 21“ von Rio aus. Einzelne EU-Mitgliedsstaaten widmeten sich produktbezogenen Fragen, wie beispielsweise Schweden, die Niederlande oder Dänemark. Auch die OECD nahm sich in einem Bericht dem Thema an (4).

- Diskussionsforen: An- und Einsichten, Erfahrungen und Konzepte wurden weltweit bei den „Green goods"-Tagungen ausgetauscht. Bisher fanden fünf dieser Tagungen statt.

\section{Der Durchbruch}

Den wesentlichen Impuls zur Fortentwicklung hat indes die Umwelt-Generaldirektion der EU gegeben. Sie bauftragte die Unternehmensberater Ernst\&Young und die Universität von Sussex, einen ersten konzeptionellen Entwurf für eine gemeinsame europäische IPP vorzubereiten. Der Bericht wurde 1998 vorgelegt und löste eine intensive Diskussion aus. Im Dezember 1998 fand in Brüssel ein Workshop statt, auf dem mehr als 180 Personen aus Politik, Verbänden, Unternehmen, NGOs und Wissenschaft über das Thema IPP diskutierten (5).

Gleichzeitig entschied sich das deutsche Bundesumweltministerium (BMU) noch unter
Angela Merkel dazu, IPP zu einem Schwerpunkt seiner EU-Ratspräsidentschaft in der ersten Jahreshälfte $1999 \mathrm{zu}$ machen. Dazu wurde im Dezember 1998 das IÖW vom BMU beauftragt, es bei der Abfassung von Hintergrunddokumenten und bei der Erarbeitung einer deutschen Konzeption zu unterstïtzen. Auf dem informellen EU-Umweltministerrat im Mai in Weimar wurde das Thema IPP auf die politische Agenda gesetzt und eine Reihe von Maßnahmen auf EU-Ebene angeregt. Ein unter wesentlicher Mitwirkung des IÖW vom BMU vorgelegtes Hintergrundpapier bildet mittlerweile den „Common ground“ in der EU (6).

\section{Wesentliche Merkmale von IPP}

Die Definition von IPP verweist auf eine gestaltende und tragende Rolle des Staates, der Rahmenbedingungen setzt, gestaltet und Einfluss nimmt. Diese Rolle ist eindeutig abzugrenzen von den Aktivitäten der Wirtschaft, anderer Akteure und Anspruchsgruppen. Deren Tätigkeiten - Produktmanagement - kommt eine konstitutive Bedeutung im Rahmen des Wechselspiels zwischen Politik und Gestaltungsund Wahlfreiheiten zu. IPP nutzt und bündelt die Kräfte des Marktes für eine ökologische Transformation im Sinne der Förderung einer nachhaltigen Entwicklung. Mit IPP hat sich eine neue ganzheitliche Betrachtung von Produkten herausgebildet. Sie interessiert sich nicht nur für das Produkt selbst, sondern für seine Einflüsse auf die Umwelt während seines gesamten Lebenszyklusses, sozusagen von der Wiege bis zur Bahre. Hier wird auch deutlich, dass mit der Betrachtung von Produktlebenszyklen eine Integration von Umweltpolitik in andere Politikbereiche angestrebt wird.

IPP ist also keine völlig neue umweltpolitische Maßnahme und keine zusätzliche umweltpolitische Verordnung, sondern stellt vielmehr eine neue Sichtweise in der Umweltpolitik dar. Das Schlüsselwort lautet in diesem Zusammenhang "Integration“. IPP ist ein in dreierlei Hinsicht ,integrierter".Ansatz:

1. In analytischer Hinsicht betrachtet IPP den gesamten Lebenszyklus von Gütern und Dienstleistungen. Das beinhaltet, dass die bedeutsamsten Einwirkungen auf die Umwelt beriicksichtigt werden und dass Verschiebungen dieser Einwirkungen zwischen einzelnen Phasen des Lebenszyklus oder von einem Umweltmedium zum nächsten erkannt werden sollen und für Abhilfe gesorgt wird. 
2. Hinsichtlich der Implementierung zielt IPP auf zahlreiche sich ergänzende Instrumente (freiwillige, informatorische, ökonomische oder auch regulierende). Diese werden je nach Anwendungskontext ausgewählt, um die gewünschten Umweltverbesserungen zu erzielen.

3. Hinsichtlich der europäischen Politik ist der produktbezogene Umweltschutz ein signifikantes Beispiel dafür, wie nachhaltige Entwicklung durch die Integration verschiedenster umweltrelevanter Politikbereiche vorangetrieben werden kann (z.B. Industrie, Verkehr, Forschung/Entwicklung, Binnenmarkt und Verbraucherschutz).

\section{Wie geht es weiter?}

Die beachtliche zeitliche Dynamik darf nicht über den erheblichen Diskussionsbedarf zwischen den Akteuren über über das Thema IPP hinwegtäuschen. Seitens der Wirtschaft werden beträchtliche Vorbehalte gegen IPP geäußert, beispielsweise bei einer Anhörung der CDU/CSU-Bundestagsfraktion am 15. April 1999. Es geht um nichts geringeres als um eine Annäherung im Verständnis von Industrie und Staat bzw. um eine Modernisierung der Umwelt- politik. Dabei geht es zum einen um konkrete wirtschaftliche Interessen sowie zum anderen aber auch darum, dass Konzept und Philosophie der IPP eben doch für die praktische Umsetzung zunächst sehr vage geblieben sind. „,̈bersetzungs-" und „Transmissions" arbeit ist gefragt. Daneben muss IPP an greifbaren Beispielen verständlich gemacht werden und das Zusammenwirken verschiedener Akteure im Sinne einer Aufgabenteilung deutlicher werden.

Solange eine gemeinsame Sprache und eine Verständigung über grundlegende Prinzipien nicht vorhanden ist, werden alte Schubladen und Lösungskonzepte nach wie vor reichlich bedient. Die Arbeit an der IPP macht bereits im Vorfeld die ständige Notwendigkeit einer Zusammenarbeit zwischen Akteuren deutlich. In diesem Zusammenhang stehen in den nächsten Monaten zwei sehr wichtige Meilensteine an: Zum einen ein Workshop zu IPP, den das BMU und der Bundesverband der Deutschen Industrie gemeinsam im Februar 2000 in Berlin veranstalten werden. Zum anderen beabsichtigt die EU-Kommission ein Grünbuch zu IPP vorzugelegen, das die Diskussion um die weitere
Implementierung strukturieren hilft. Produkte sind Globetrotter - gerade deshalb ist die EU gefordert.

\section{Anmerkungen}

(1) BMU: Umwelt, Heft 6/1999, S. V

(2) Vgl. Rubik, F./ G. Scholl: The Ripening of Integrated Product Policy. In: European Environment, Nr. 9/1999.

(3) Oosterhuis, F./ F. Rubik/ G. Scholl: Product Policy in Europe. New Environmental Perspectives, Dordrecht et al. 1996.

(4) OECD: Preliminary results of (Sustainable) Product

Policy Survey. Paris.

(5) Die Proceedings des Workshops finden sich unter http://europa.eu.int/comm/dg11/ipp/home.htm

(6) Zu Hintergrundpapier und Schlussfolgerungen des Umweltministerrates vgl. BMU: Umwelt, Heft Nr. 6/1999.

\section{Die Autorlnnen}

Frieder Rubik und Dr. Ulla Simshäuser sind wissenschaftliche Milorbeilerlnnen des IÖW im Regional. büro Baden-Württemberg.

Kontakt: I0̈W, Bergstraße 7, 69120 Heidelberg, Tel. 06221/ 64916-0, Fax 06221/ 27060,

E-mail: mailbox@heidelberg.ioew.de

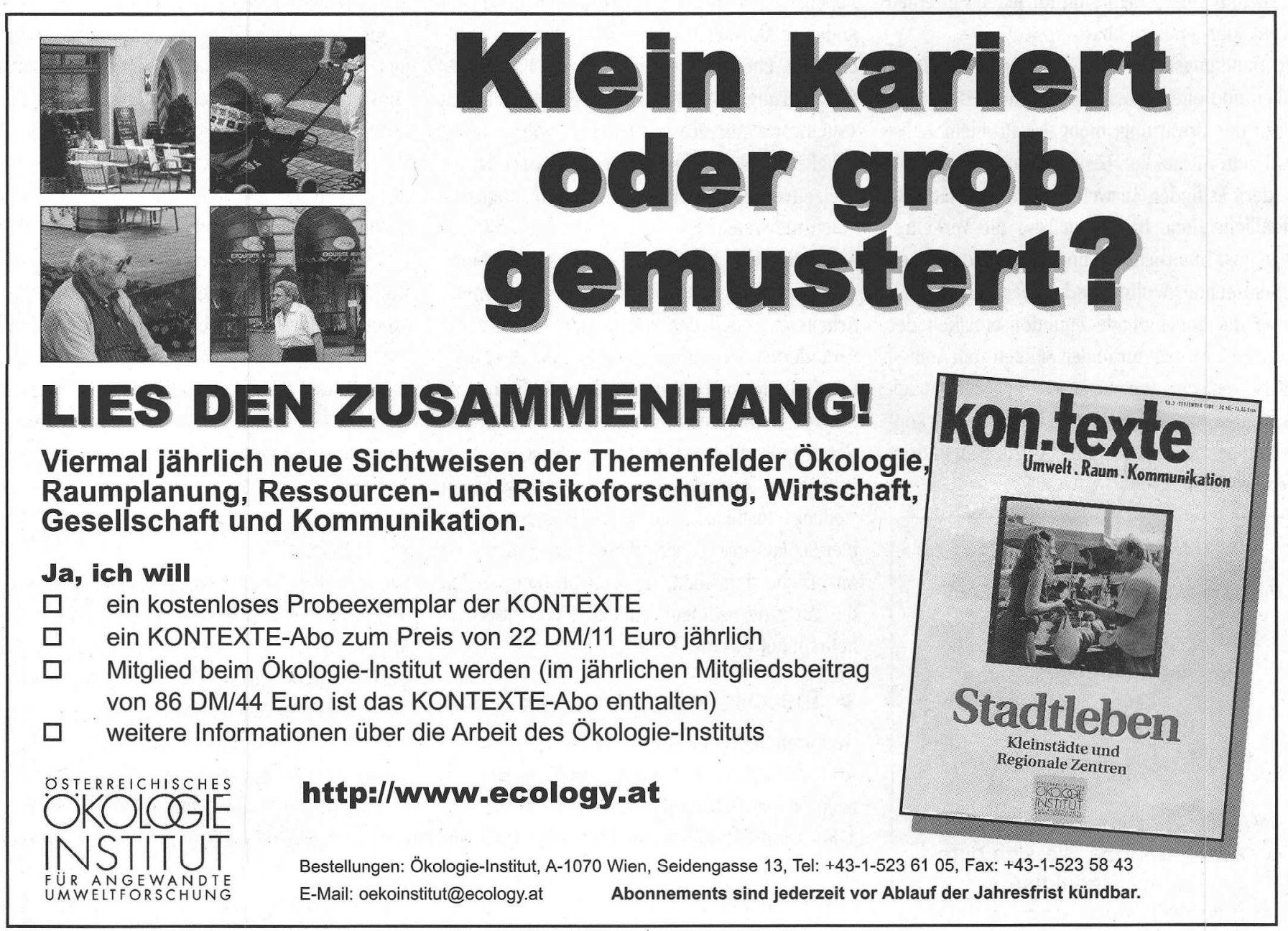


(c) 20I0 Authors; licensee IÖW and oekom verlag. This is an article distributed under the terms of the Creative Commons Attribution Non-Commercial No Derivates License (http://creativecommons.org/licenses/by-nc-nd/3.o/), which permits unrestricted use, distribution, and reproduction in any medium, provided the original work is properly cited. 